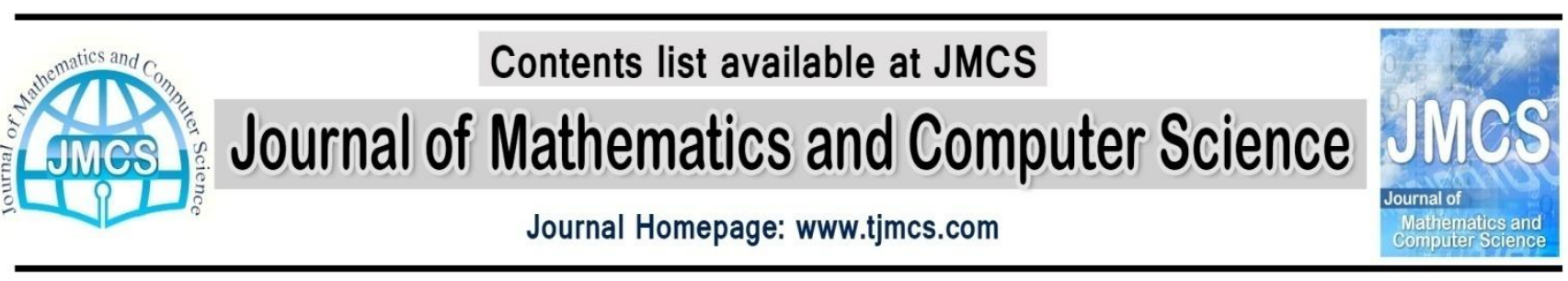

\title{
A nonlinear partial integro-differential equation arising in population dynamic via radial basis functions and theta-method
}

\author{
Mohammad Aslefallah $^{1,2}$ and Elyas Shivanian ${ }^{1}$ \\ ${ }^{1}$ Department of Mathematics, Imam Khomeini International University, Qazvin, Iran \\ ${ }^{2}$ Department of Mathematics, Karaj Branch, Islamic Azad University, Karaj, Iran
}

Article history:

shivanian@sci.ikiu.ac.ir,

Received July 2014

Accepted August 2014

Available online September 2014

\begin{abstract}
This paper proposes a numerical method to deal with the integro-differential reaction-diffusion equation. In the proposed method, the time variable is eliminated by using finite difference $\theta-$ method to enjoy the stability condition. The method benefits from collocation radial basis function method, the generallized thin plate splines (GTPS) radial basis functions are used. Therefore, it does not require any struggle to determine shape parameter. The obtained results for some numerical examples reveal that the proposed technique is very effective, convenient and quite accurate to such considered problems.
\end{abstract}

Keywords: Integro-differential equation, Radial basis functions, Kansa method, Finite differences $\theta-$ method.

2010 Mathematics subject classification: 34A08,35R11,65M06.

\section{Introduction}

Many problems in science and engineering modelled as differential equations. Solving equations by traditional numerical methods such as finite difference (FDM), finite element (FEM) needs generation of a regular mesh in the domain of the problem which is computationally expensive $[1,2,3,4,5]$. During the last decade, meshless methods have received much attention. Due to the difficulty of the mesh generation problem, meshless methods for simulation of the numerical problems 
are employed. Radial basis functions (RBFs) interpolation is a technique for representing a function starting with data on scattered points $[6,7,8,9]$. The RBFs can be of various types, such as: polynomials of a given degree; linear, quadratic, cubic, etc; thin plate spline (TPS), multiquadrics (MQ), inverse multiquadrics (IMQ), Gaussian forms (GA), etc. Most differential equations do not have exact analytic solutions, so approximation and numerical techniques must be used.

Development of constructive methods for the numerical solution of mathematical problems is a main branch of mathematics. Meshless methods have attracted much attention in the both mathematics and engineering community, recently. Extensive developments have been made in several varieties of meshless techniques and applied to many applications in science and engineering. These methods exist under different names, such as: the diffuse element method (DEM) [10], the hp-cloud method [11], Meshless Local Petrov- Galerkin (MLPG) method [12,13,14], the meshless local boundary integral equation (LBIE) method [15], the partition of unity method (PUM) [16], the meshless collocation method based on radial basis functions (RBFs)[17], the smooth particle hydrodynamics (SPH)[19], the reproducing kernel particle method (RKPM) [20], the radial point interpolation method [22], meshless local radial point interpolation method (MLRPI) [23,24], and so on.

In this study, we implement the meshless collocation method for solving the following integrodifferential reaction-diffusion equation (also arising in population dynamic) $[25,26]$ by using a radial basis function (RBF):

$$
\frac{\partial u(x, t)}{\partial t}=\frac{\partial^{2} u(x, t)}{\partial x^{2}}+\beta u(1-a u-b J(x, t))+f(x, t)
$$

where:

$$
J(x, t)=\int_{x_{L}}^{x_{R}} \psi(x-y) u(y, t) d y
$$

(for $t \in[0, T]$ ) on a finite domain $x_{L}<x<x_{R} . \psi(x)$ is kernel function and $f(x, t)$ is a given smooth function.

Initial condition $u(x, 0)=g(x)$ for $x_{L}<x<x_{R}$ and boundary conditions are as follows: $u\left(x_{L}, t\right)=0$ and $u\left(x_{R}, t\right)=0$.

In the special case, if $b=0$ and $f(x, t)=0$ we have well-known Fisher's equation as:

$$
\frac{\partial u(x, t)}{\partial t}=\frac{\partial^{2} u(x, t)}{\partial x^{2}}+\beta u(1-a u)
$$

\section{Preliminaries}

For implementation of this method we need the following definitions.

Definition 2.1 (Radial basis functions.) Considering a finite set of interpolation points $X=\left\{x_{1}, x_{2}, \ldots, x_{M}\right\} \subseteq R^{d}$ and a function $u: X \rightarrow R^{d}$, according to the process of interpolation using radial basis functions [6], the interpolant of $u$ is constructed in the following form: 


$$
(S u)(x)=\sum_{i=1}^{M} \lambda_{i} \varphi\left(\left\|x-x_{i}\right\|\right)+p(x), \quad x \in R^{d}
$$

where $\|$.$\| is the Euclidean norm and \varphi(\|\|$.$) is a radial function. Also, p(x)$ is a linear combination of polynomials on $R^{d}$ of total degree at most $m-1$ as follows:

$$
p(x)=\sum_{j=M+1}^{M+l} \lambda_{j} q_{j}(x), \quad l=\left(\begin{array}{c}
m+d-1 \\
d
\end{array}\right)
$$

Moreover, the interpolant $S u$ and additional conditions must be determined to satisfy the system:

$$
\begin{cases}(S u)\left(x_{i}\right)=u\left(x_{i}\right) & , \quad i=1,2, \ldots, M \\ \sum_{i=1}^{M} \lambda_{i} q_{j}\left(x_{i}\right)=0, & , \quad \forall q_{j} \in \Pi_{m-1}^{d}\end{cases}
$$

where $\Pi_{m-1}^{d}$ denotes the space of all polynomials on $R^{d}$ of total degree at most $m-1$. Now we have a unique interpolant $(S u)$ of $\mathrm{u}$ if $\varphi(r)$ is a conditionally positive definite radial basis function of order $\mathrm{m}[28]$. For any partial differential operator $L, L u$ can be represented by:

$$
L u(x)=\sum_{x_{i} \in X} \lambda_{i} L \varphi\left(\left\|x-x_{i}\right\|\right)+L p(x),
$$

The coefficients $\lambda_{i}$ will be obtained by solving the system of linear equations.

We will use some RBFs which have the following form:

$$
\varphi\left(\left\|x-x_{i}\right\|\right)=\varphi\left(r_{i}\right)
$$

Some types of RBFs presented in Table.1. (c is shape parameter)

Table 1. Some types of RBF functions

\begin{tabular}{|c|c|c|}
\hline Name & Abbreviation & Formula \\
\hline Cubic & CU & $\varphi(r)=r^{3}$ \\
\hline Thin plate splines & TPS & $\varphi(r)=r^{2} \log (r)$ \\
\hline Generalized Thin plate splines & GTPS & $\varphi(r)=r^{2 m} \log (r), \quad m \in N$ \\
\hline Inverse quadrics(or Cauchy) & IQ & $\varphi(r)=\frac{1}{c^{2}+r^{2}}$ \\
\hline Multiquadrics & MQ & $\varphi(r)=\sqrt{c^{2}+r^{2}}$ \\
\hline Inverse Multiquadrics & IMQ & $\varphi(r)=\frac{1}{\sqrt{c^{2}+r^{2}}}$ \\
\hline Gaussian RBF & GA & $\varphi(r)=e^{-r^{2} / c^{2}}$ \\
\hline
\end{tabular}

Definition $2.2 \theta$-method, $(0 \leq \theta \leq 1)$, is general finite-difference approximation to $\frac{\partial^{2} u(x, t)}{\partial x^{2}}$ given by:

$$
\frac{\partial^{2} u(x, t)}{\partial x^{2}} \cong \theta \delta_{2, x} U_{i, j+1}+(1-\theta) \delta_{2, x} U_{i, j}
$$

such that we define: 


$$
\nabla^{2}=\delta_{2, x} U_{i, j}=\frac{1}{(\Delta x)^{2}}\left(U_{i+1, j}-2 U_{i, j}+U_{i-1, j}\right)
$$

(where $h=\Delta x=\frac{x_{R}-x_{L}}{M}$ for $x$-axis and $U_{i, j}=U_{i}^{j}=U\left(x_{i}, t_{j}\right)$ represent the numerical approximation solution) In other words:

$$
\frac{\partial^{2} u(x, t)}{\partial x^{2}} \cong \frac{1}{(\Delta x)^{2}}\left\{\theta\left(U_{i+1, j+1}-2 U_{i, j+1}+U_{i-1, j+1}\right)+(1-\theta)\left(U_{i+1, j}-2 U_{i, j}+U_{i-1, j}\right)\right\},
$$

Remark 2.3 Note that $\theta=0$ gives the explicit scheme, $\theta=\frac{1}{2}$ the Crank-Nicolson, and $\theta=1$ a fully implicit backward time-difference method.

Remark 2.4 The laplacian operator $\nabla^{2}$ for $\varphi$ function is given by

$$
\nabla^{2}(\varphi(r))=\frac{\partial \varphi}{\partial r}\left(\frac{\partial^{2} r}{\partial x^{2}}\right)+\frac{\partial^{2} \varphi}{\partial r^{2}}\left(\frac{\partial r}{\partial x}\right)^{2}
$$

\section{Discretization}

According to definitions (2.1)and (2.2), from (1) and $\theta$-method we get:

$$
\frac{\partial u\left(x, t^{n+1}\right)}{\partial t}=\left[\theta \nabla^{2} u^{n+1}+(1-\theta) \nabla^{2} u^{n}\right]+\beta u^{n+1}-a \beta\left(u^{n}\right)^{2}-b \beta u^{n} J^{n}+f^{n+1},
$$

By substituting finite difference for left hand into (4) we have:

$$
\frac{u^{n+1}-u^{n}}{\Delta t}=\left[\theta \nabla^{2} u^{n+1}+(1-\theta) \nabla^{2} u^{n}\right]+\beta u^{n+1}-a \beta\left(u^{n}\right)^{2}-b \beta u^{n} J^{n}+f^{n+1},
$$

and for $\Delta t=k$ :

$$
u^{n+1}-k \theta \nabla^{2} u^{n+1}-k \beta u^{n+1}=u^{n}+k(1-\theta) \nabla^{2} u^{n}-k a \beta\left(u^{n}\right)^{2}-k b \beta u^{n} J^{n}+k f^{n+1},
$$

In other words, we get:

$$
\left(1-k \theta \nabla^{2}-k \beta\right) u_{i}^{n+1}=u_{i}^{n}+k(1-\theta) \nabla^{2} u_{i}^{n}-k a \beta\left(u_{i}^{n}\right)^{2}-k b \beta u_{i}^{n} J_{i}^{n}+k f_{i}^{n+1},
$$

Now, according to the mentioned method in one-dimensional case, if we collocate $M$ different points $x_{1}, x_{2}, \ldots, x_{M}$, then:

$$
u\left(x_{i}, t_{n+1}\right)=\sum_{j=1}^{M} \lambda_{j}^{n+1} \varphi\left(\left\|x_{i}-x_{j}\right\|\right)+\lambda_{M+1}^{n+1} x_{i}+\lambda_{M+2}^{n+1},
$$

Two additional conditions can be described as:

$$
\sum_{j=1}^{M} \lambda_{j}^{n+1}=\sum_{j=1}^{M} \lambda_{j}^{n+1} x_{i}=0,
$$

Finally, by combining equations (8),(9), we obtain a matrix form:

$$
[u]^{n+1}=A[\lambda]^{n+1}
$$


where: $[u]^{n+1}=\left[u_{1}^{n+1}, u_{2}^{n+1}, \ldots, u_{M}^{n+1}, 0,0\right]^{T} \quad, \quad[\lambda]^{n+1}=\left[\lambda_{1}^{n+1}, \lambda_{2}^{n+1}, \ldots, \lambda_{M+2}^{n+1}\right]^{T}$ and the matrix $A=\left(a_{i j}\right)_{(M+2) \times(M+2)}$ is given by:

$$
A=\left[\begin{array}{lllllll}
\varphi_{11} & \cdots & \varphi_{1 j} & \cdots & \varphi_{1 M} & x_{1} & 1 \\
\vdots & \ddots & \vdots & \ddots & \vdots & \vdots & \vdots \\
\varphi_{i 1} & \cdots & \varphi_{i j} & \cdots & \varphi_{i M} & x_{i} & 1 \\
\vdots & \ddots & \vdots & \ddots & \vdots & \vdots & \vdots \\
\varphi_{M 1} & \cdots & \varphi_{M j} & \cdots & \varphi_{M M} & x_{M} & 1 \\
x_{1} & \cdots & x_{j} & \cdots & x_{M} & 0 & 0 \\
1 & \cdots & 1 & \cdots & 1 & 0 & 0
\end{array}\right]
$$

By substituting (8) into (5),(6) and considering (9) and initial and boundary conditions we obtain a matrix form:

$$
[c]^{n+1}=B[\lambda]^{n+1}
$$

where: $[c]^{n+1}=\left[c_{1}^{n+1}, c_{2}^{n+1}, \ldots, c_{M}^{n+1}, 0,0\right]^{T}$ and

$$
B=\left[\begin{array}{lllllll}
L\left(\varphi_{11}\right) & \cdots & L\left(\varphi_{1 j}\right) & \cdots & L\left(\varphi_{1 M}\right) & L\left(x_{1}\right) & L(1) \\
\vdots & \ddots & \vdots & \ddots & \vdots & \vdots & \vdots \\
L\left(\varphi_{i 1}\right) & \cdots & L\left(\varphi_{i j}\right) & \cdots & L\left(\varphi_{i M}\right) & L\left(x_{i}\right) & L(1) \\
\vdots & \ddots & \vdots & \ddots & \vdots & \vdots & \vdots \\
L\left(\varphi_{M 1}\right) & \cdots & L\left(\varphi_{M j}\right) & \cdots & L\left(\varphi_{M M}\right) & L\left(x_{M}\right) & L(1) \\
x_{1} & \cdots & x_{j} & \cdots & x_{M} & 0 & 0 \\
1 & \cdots & 1 & \cdots & 1 & 0 & 0
\end{array}\right]
$$

where $L$ represents an operator given by

$$
L(*)= \begin{cases}\left(1-k \theta \nabla^{2}-k \beta\right)(*), & 1<i<M \\ (*), & i=1 \text { or } i=M\end{cases}
$$

and

$$
c_{i}^{n+1}= \begin{cases}u^{n}+k(1-\theta) \nabla^{2} u^{n}-k a \beta\left(u^{n}\right)^{2}-k b \beta J_{i}^{n}+k f^{n+1}, & n \geq 0,1<i<M \\ g\left(x_{i}, t_{n+1}\right), & i=1 \text { or } i=M\end{cases}
$$

We use the Simpson method for approximation of the integral term. By solving the system (11) we find $(M+2)$ unknowns $\lambda_{j}^{n+1}$ then with (8) we approximate the value of $u$.

\section{Numerical Examples}

We use the generalized thin plate splines(GTPS) which have the following form: 


$$
\varphi(r)=r^{2 m} \log (r), \quad m \in N
$$

Example 4.1 Consider (1) in the special case for $a=b=0, \quad \beta=-1$ (as a linear example):

$$
\frac{\partial u(x, t)}{\partial t}=\frac{\partial^{2} u(x, t)}{\partial x^{2}}-u(x, t)+f(x, t),
$$

(for $t \in[0, T]$ ) on a finite domain $[0,2]$. Initial condition $u(x, 0)=g(x)=0$ and boundary conditions are: $u(0, t)=u(2, t)=0$.

with an exact analytical solution:

$$
u(x, t)=t^{2} x(2-x) . \quad 0 \leq x \leq 2
$$

when:

$$
f(x, t)=2 t x(2-x)+t^{2} x(2-x)+2 t^{2}
$$

For $m=4$ in GTPS, the approximated solution and error functions shown in Fig. 1 verify the reliability of presented method. The Root mean square(RMS) of error value for some different values of $\theta$ and $\mathrm{M}$ are given in Table2.

Table 2. Root mean square(RMS) of Example 4.1 at time $T=2$.

\begin{tabular}{|c|c|c|c|}
\hline$\theta$ & $\mathrm{M}$ & $\Delta t$ & $\mathrm{RMS}$ \\
\hline 0.5 & 10 & 0.10 & $3.2745 \mathrm{e}-3$ \\
\hline & 20 & 0.05 & $9.3012 \mathrm{e}-4$ \\
\hline & 50 & 0.02 & $2.2047 \mathrm{e}-4$ \\
\hline & 100 & 0.01 & $9.2689 \mathrm{e}-5$ \\
\hline 0.75 & 10 & 0.10 & $2.1991 \mathrm{e}-3$ \\
\hline & 20 & 0.05 & $6.8368 \mathrm{e}-4$ \\
\hline & 50 & 0.02 & $1.9322 \mathrm{e}-4$ \\
\hline & 100 & 0.01 & $8.8321 \mathrm{e}-5$ \\
\hline 1 & 10 & 0.10 & $1.2820 \mathrm{e}-3$ \\
\hline & 20 & 0.05 & $4.9447 \mathrm{e}-4$ \\
\hline & 50 & 0.02 & $1.7504 \mathrm{e}-4$ \\
\hline & 100 & 0.01 & $8.5429 \mathrm{e}-5$ \\
\hline
\end{tabular}



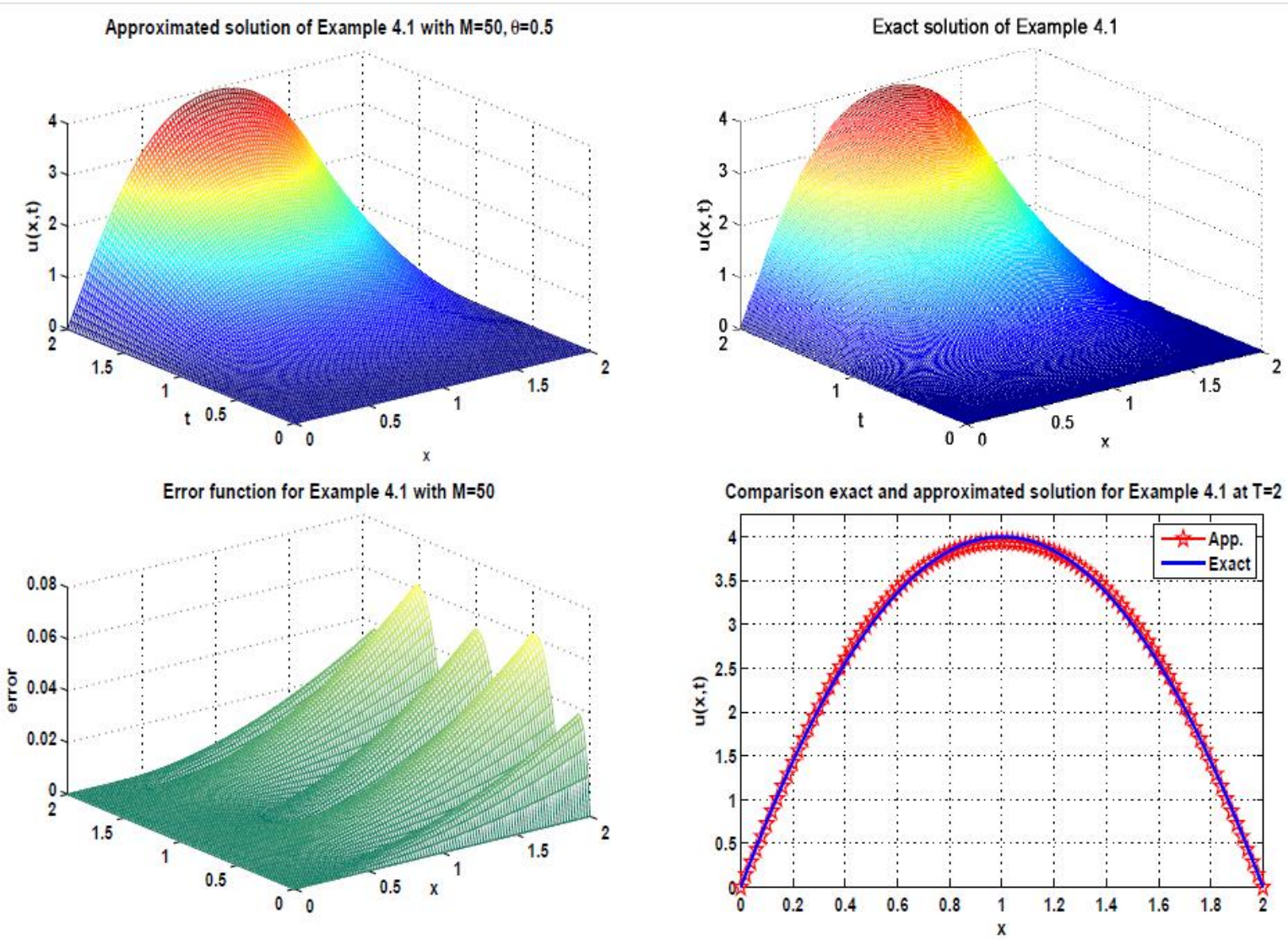

Figure 1. Comparison between exact and numerical solutions for the Example 4.1 with $M=50, \theta=0.5$

Example 4.2 Consider (1) in the special case for $a=1, b=0, \quad \beta=1$ (as a nonlinear example):

$$
\frac{\partial u(x, t)}{\partial t}=\frac{\partial^{2} u(x, t)}{\partial x^{2}}+u(1-u)+f(x, t),
$$

(for $t \in[0, T]$ ) on a finite domain $[-1,1]$. Initial condition $u(x, 0)=g(x)=\left(1-x^{4}\right) \sin (x)$ and boundary conditions are: $\quad u(-1, t)=u(1, t)=0$.

with an exact analytical solution:

$$
u(x, t)=\left(1-x^{4}\right) \sin (x+t) . \quad-1 \leq x \leq 1
$$

when:

$$
f(x, t)=\left(1+8 x^{3}-x^{4}\right) \cos (x+t)+12 x^{2} \sin (x+t)+\left(1-x^{4}\right)^{2} \sin ^{2}(x+t),
$$

For $m=4$ in GTPS, the approximated solution and error functions shown in Fig. 2 verify the reliability of presented method. The Root mean square(RMS) of error value for some different values of $\theta$ and $M$ are given in Table3. 
Table 3. Root mean square(RMS) of Example 4.2 at time $T=2$.

\begin{tabular}{|c|c|c|c|}
\hline$\theta$ & $\mathrm{M}$ & $\Delta t$ & RMS \\
\hline 0.5 & 10 & 0.10 & $1.7312 \mathrm{e}-3$ \\
\hline & 20 & 0.05 & $4.7132 \mathrm{e}-4$ \\
\hline & 50 & 0.02 & $9.1033 \mathrm{e}-5$ \\
\hline & 100 & 0.01 & $3.1253 \mathrm{e}-5$ \\
\hline 0.75 & 10 & 0.10 & $9.9762 \mathrm{e}-4$ \\
\hline & 20 & 0.05 & $2.8700 \mathrm{e}-4$ \\
\hline & 50 & 0.02 & $6.5481 \mathrm{e}-5$ \\
\hline & 100 & 0.01 & $2.6401 \mathrm{e}-5$ \\
\hline & 10 & 0.10 & $5.1844 \mathrm{e}-4$ \\
\hline & 20 & 0.05 & $1.6413 \mathrm{e}-4$ \\
\hline & 50 & 0.02 & $4.9833 \mathrm{e}-5$ \\
\hline & 100 & 0.01 & $2.3557 \mathrm{e}-5$ \\
\hline
\end{tabular}
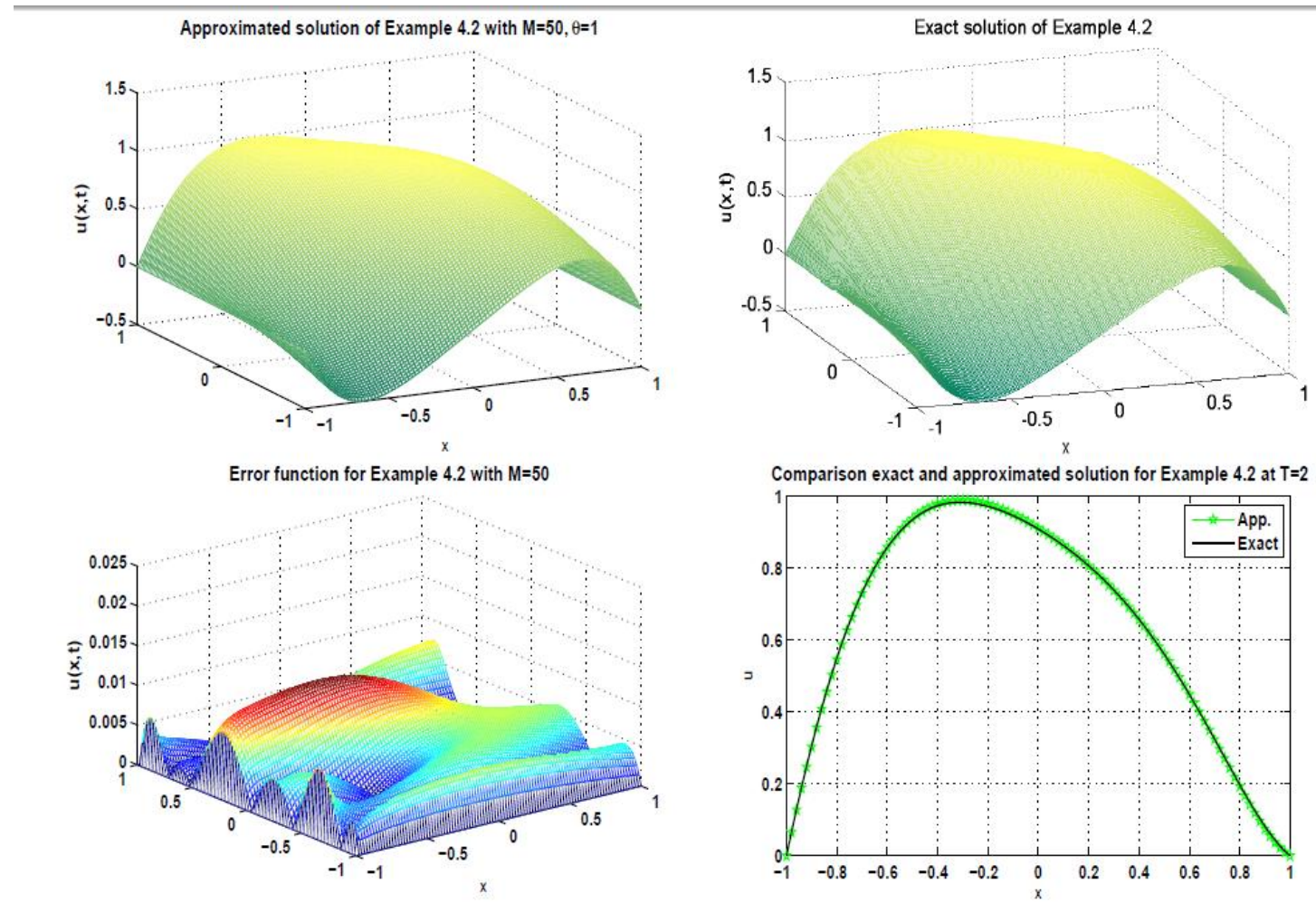

Figure 2. Comparison between exact and numerical solutions for the Example 4.2 with $M=50, \theta=0.5$

Example 4.3 Consider (1) for $a=1, b=1, \quad \beta=1$ (as a nonlinear integro-differential example) :

$$
\frac{\partial u(x, t)}{\partial t}=\frac{\partial^{2} u(x, t)}{\partial x^{2}}+u(1-u-J(x, t))+f(x, t),
$$

(for $t \in[0, T]$ ) on a finite domain $[-1,1]$.

Where: 


$$
J(x, t)=\int_{-1}^{1} e^{x-y} u(y, t) d y
$$

Initial condition $u(x, 0)=g(x)=\sin (2 \pi x)$ and boundary conditions are: $\quad u(-1, t)=u(1, t)=0$.

with an exact analytical solution:

$$
u(x, t)=\left(1-x^{4}\right) \sin (x+t) . \quad-1 \leq x \leq 1
$$

when:

$$
f(x, t)=u(x, t) J(x, t)+h(x, t)
$$

and:

$$
h(x, t)=\left(1+8 x^{3}-x^{4}\right) \cos (x+t)+12 x^{2} \sin (x+t)+\left(1-x^{4}\right)^{2} \sin ^{2}(x+t),
$$

For $m=3$ in GTPS, the approximated solution and error functions shown in Fig. 3 verify the reliability of presented method. The Root mean square(RMS) of error value for some different values of $\theta$ and $\mathrm{M}$ are given in Table4.

Table 4. Root mean square(RMS) of Example 4.3 at time $T=2$.

\begin{tabular}{|c|c|c|c|}
\hline$\theta$ & $\mathrm{M}$ & $\Delta t$ & RMS \\
\hline 0.5 & 10 & 0.10 & $1.8278 \mathrm{e}-3$ \\
\hline & 20 & 0.05 & $6.2988 \mathrm{e}-4$ \\
\hline & 50 & 0.02 & $2.0270 \mathrm{e}-4$ \\
\hline & 100 & 0.01 & $9.7285 \mathrm{e}-5$ \\
\hline 0.75 & 10 & 0.10 & $1.1707 \mathrm{e}-3$ \\
\hline & 20 & 0.05 & $5.0246 \mathrm{e}-4$ \\
\hline & 50 & 0.02 & $1.9135 \mathrm{e}-4$ \\
\hline & 100 & 0.01 & $9.5507 \mathrm{e}-5$ \\
\hline 1 & 10 & 0.10 & $8.0165 \mathrm{e}-4$ \\
\hline & 20 & 0.05 & $4.3730 \mathrm{e}-4$ \\
\hline & 50 & 0.02 & $1.8530 \mathrm{e}-4$ \\
\hline & 100 & 0.01 & $9.4433 \mathrm{e}-5$ \\
\hline
\end{tabular}



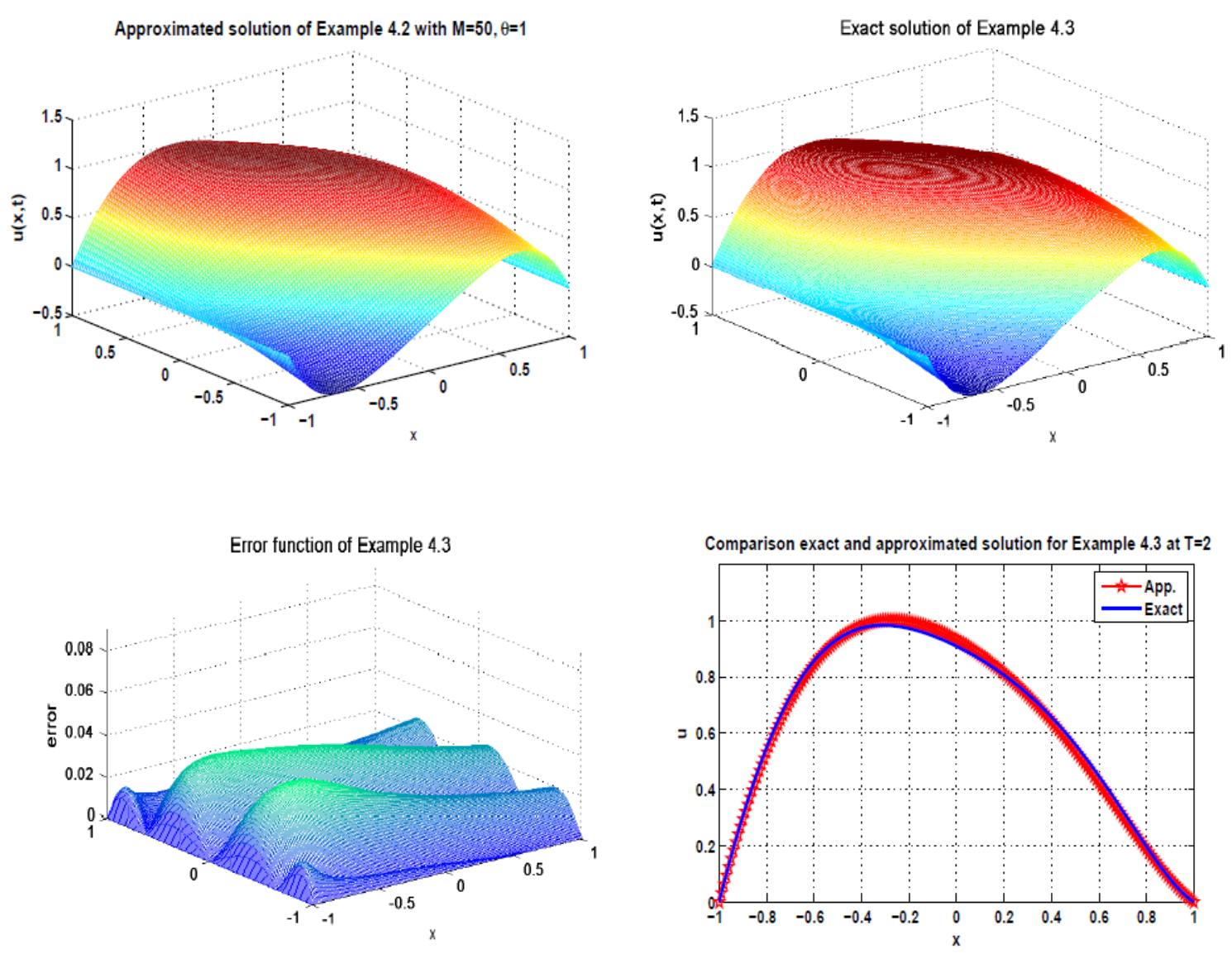

Figure 3. Comparison between exact and numerical solutions for the Example 4.3 with $M=50, \theta=0.5$

\section{Conclusion}

In this paper we have presented a numerical scheme based on combination of meshless collocation radial basis function (so-called Kansa's method) and $\theta$-method. The $\theta$-method has been applied to derivative. The method has been tested on three illustrative numerical examples. The computational results are found to be in good agreement with the exact solutions.

\section{References}

[1] L. Sabatelli, S. Keating, J. Dudley, P. Richmond: Waiting time distributions in financial markets, European Physical Journal B, 27, (2002), 273-275.

[2] R. Schumer, D.A. Benson, M.M. Meerschaert, S.W. Wheatcraft: Eulerian derivation of the fractional advection-dispersion equation, Journal of Contaminant Hydrology, 48, (2001), 69-88.

[3] R. Hilfer: Applications of Fractional Calculus in Physics, World Scientific, Singapore. (2000) 
[4] M.M. Meerschaert, C. Tadjeran: Finite Difference Approximations for two-sided spacefractional partial differential equations, Applied Numerical Mathematics, 56, (2006), 80-90.

[5] S. Samko, A. Kibas, O. Marichev: Fractional Integrals and derivatives: Theory and Applications, Gordon and Breach, London. (1993)

[6] MD. Buhmann: Radial basis functions: theory and implementations. Cambridge University Press. (2003)

[7] V.R. Hosseini, W. Chen, Z. Avazzadeh: Numerical solution of fractional telegraph equation by using radial basis functions, Eng. Anal. Boundary. Elem. ,38, (2014), 31-39.

[8] S. Abbasbandy, H.R. Ghehsareh, I. Hashim: Numerical analysis of a mathematical model for capillary formation in tumor angiogenesis using a meshfree method based on the radial basis function, Eng. Anal. Boundary. Elem. 36(12), (2012), 1811-1818.

[9] S. Abbasbandy, H.R. Ghehsareh, I. Hashim: A meshfree method for the solution of twodimensional cubic nonlinear schrödinger equation, Eng. Anal. Boundary. Elem. 37(6), (2013), 885-898.

[10] B. Nayroles, G. Touzot, P. Villon: Generalizing the finite element method: diffuse approximation and diffuse elements, Comput. Mech., 10, (1992), 307-318.

[11] C. Duarte, J. Oden: An h-p adaptative method using clouds, Comput. Methods Appl. Mech. Engrg. 139, (1996), 237-262.

[12] S.N. Atluri, T.L. Zhu: A new meshless local Petrov-Galerkin (MLPG) approach in computational mechanics, Comput. Mech. 22 (2), (1998), 117-127.

[13] S. Abbasbandy, V. Sladek, A. Shirzadi, J. Sladek: Numerical Simulations for Coupled Pair of Diffusion Equations by MLPG Method, CMES Compt. Model. Eng. Sci. 71 (1), (2011), 15-37.

[14] A. Shirzadi, L. Ling, S. Abbasbandy: Meshless simulations of the two-dimensional fractionaltime convectiondiffusion- reaction equations, Eng. Anal. Bound. Elem. 36, (2012), 1522-1527.

[15] T. Zhu, J.D. Zhang, S.N. Atluri: A local boundary integral equation (LBIE) method in computational mechanics and a meshless discretization approach, Comput. Mech. 21, (1998), 223-235.

[16] J.M. Melenk, I. Babŭska: The partition of unity finite element method: basic theory and applications, Comput. Methods Appl. Mech. Engrg. 139, (1996), 289-314.

[17] E.J. Kansa: Multiquadrics-a scattered data approximation scheme with applications to computational fluid dynamics-II, J. Comput. Math. Appl. 19, (1990), 147-161.

[18] M. Aslefallah, D. Rostamy: A Numerical Scheme For Solving Space-Fractional Equation By Finite Differences Theta-Method, Int. J. of Adv. in Aply. Math. and Mech., 1(4) , (2014),1-9.

[19] L.B. Lucy: A numerical approach to the testing of fusion process, Astron. J. 88, (1977), 1013-1024. 
[20] W.K. Liu, S. Jun, Y.F. Zhang: Reproducing kernel particle methods, Int. J. Numer. Methods Fluids, 21, (1995), 1081-1106.

[21] M. Aslefallah, D. Rostamy, K. Hosseinkhani: Solving time-fractional differential diffusion equation by theta-method, Int. J. of Adv. in Aply. Math. and Mech., 2(1) , (2014),1-8.

[22] G.R. Liu: Mesh Free Methods: Moving beyond the Finite Element Method, CRC Press.(2003)

[23] E. Shivanian: Analysis of meshless local radial point interpolation (MLRPI) on a nonlinear partial integro-differential equation arising in population dynamics, Eng. Anal. Boundary. Elem. 37, (2013), 1693-1702.

[24] M. Dehghan, A. Ghesmati: Numerical simulation of two-dimensional sine-gordon solitons via a local weak meshless technique based on the radial point interpolation method (RPIM), Comput. Phys. Commun. 181, (2010), 772-786.

[25] N. Apreutesei, N. Bessonov, V. Volpert, V. Vougalter: Spatial structures and generalized travelling waves for an integro-differential equation, Discrete and Continuous Dynamical Systems Series B, 13, (2010), 537-557.

[26] F. Fakhar-Izadi, M. Dehghan: An efficient pseudo-spectral LegendreGalerkin method for solving a nonlinear partial integro-differential equation arising in population dynamics. Math. Methods Appl. Sci. 36(12), (2013), 1485-1511.

[27] M. Aslefallah, D. Rostamy: Numerical solution for Poisson fractional equation via finite differences theta-method, J. Math. Computer Sci. , TJMCS, 12(2) , (2014), 132-142.

[28] C.A. Micchell: Interpolation of scattered data; distance matrices and conditionally positive definite functions, Constr. Approx., 2, (1986), 11-22. 